\title{
Cxcl16 Interact With SARS-CoV N Protein In and Out Cell
}

\author{
Yuan-peng ZHANG ${ }^{1}$, Rong-wu ZHANG ${ }^{2}$, Wei-shan $\mathrm{CHANG}^{1{ }^{* * *}}$ and Yan-yanWANG ${ }^{1}$ \\ (1. College of Animal Science and Technology, Shandong Agricultural University, Tai'an 271018, China; 2. Ling \\ County Animal Husboundry Bureall,Dezhou 253500, China)
}

\begin{abstract}
Our study investigated the host cell protein which can interact with SARS-CoV N protein, and explored the functional connections. The eukaryotic expression vectors pEGFP-N1/SARS-CoVN and pdsRed2-N1/ CXCL16 were constructed and used to co-transfect HEK293FT cells by the calcium phosphate method. The HIS-tagged fusion protein SARS-CoVN-GFP was then built and purified for the binding assay in vitro. The co-localization of SARS-CoVN and CXCL16 in the cytoplasm of HEK293FT cells was also shown using confocal laser scanning microscopy. It is suggested that their interaction might be through direct combination. Under a fluorescence microscope, it was observed that the purified fusion protein SARS-CoVN-GFP was attached to the cell membrane of CXCL16-transfected cells, indicating that SARS-CoVN and CXCL16 can be mutually combined.
\end{abstract}

Key words: SARS-CoVN; CXCL16; Eukaryotic expression; Confocal laser scanning

SARS coronavirus (SARS-CoV) has been identified as an etiologic agent of Severe Acute Respiratory Syndrome. Its genome consists of a positive strand RNA with a length of 30,000 nucleotides, which contains 10 overlapping Open Reading Frames (ORF) and a bilateral non-coding area. The structural proteins encoded by coronavirus include Nucleocapsid protein $(\mathrm{N})$, Envelope protein $(\mathrm{E})$, Membrane protein (M) and Spike protein (S). The multiple roles of $\mathrm{N}$ protein include participation in the formation of SARS-CoV nucleocapsid, modulation of genome RNA synthesis and transcription and translation of

\footnotetext{
Received: 2010-01-22, Accepted:2010-06-30

** Corresponding author.

Phone: +86-538-8249951-8213, Fax: +86-538-8241419.

E-mail: wschang $(a$,sdau.edu.cn
}

sub-genome RNA; it also inhibits host cell proliferation and delays the cell growth by disrupting mitosis $^{[12]}$. SARS-CoV N Protein interacts with a number of proteins within the virus or the host cells, which interferes with the cellular cycle and transforms the cellular vital activities by influencing multiple signal transduction pathways of the host cells ${ }^{[4]}$. The SARS-CoV $\mathrm{N}$ protein binding proteins identified in cells include: hnRNPA1 $1^{[7,9,11]}$, 14-3-3 $\operatorname{protein}^{[11]}$, cyclin-CDK complex ${ }^{[12]}$, human Ubiquitin binding enzyme 9 (hUbc9) ${ }^{[3]}$ and cyclophilin $\mathrm{A}^{[6]}$. Recently, $\mathrm{N}$ protein was reported to associate with Smad3 and to promote Smad3-p300 complex formation by interfering with the complex formation between $\operatorname{Smad} 3$ and $\operatorname{Smad} 4^{[15]}$. However, considering what is 
known about the host genome, the proteomics and the cellular signal transduction pathways represent only a portion of the interactors. Brunn et al investigated the interaction between SARS-CoV and host cells and discovered that the cross-reactions of the different components of the virus and the interaction between virus and host cells make up a complicated network ${ }^{[1]}$. Therefore, it is necessary to select the SARS-CoV interaction protein in the host cells with proper modalities, which in return greatly contributes to the elucidation of the pathogenesis of SARS-CoV and the search for new drug targets. Chang et al employed the SARS-CoV N protein as bait in the $\mathrm{Y} 2 \mathrm{H}$ system, and selected 15 interactors of SARS-CoV nucleocapsid from the human embryo-lung cDNA library of TwoHybridization (LLH). Finally, Immunological coprecipitation (CO-IP) was used to verify the interaction between CXCL16 and SARS-CoV N protein and that this interaction could be blocked by SARS-CoV $\mathrm{N}$ antibody ${ }^{[2]}$. In this paper we continue studying the interaction of SARS-CoV $\mathrm{N}$ and CXCL16 both in and out of the cell.

\section{MATERIALS AND METHODS}

\section{Cell and plasmids}

HEK293FT cells were grown in DMEM (Gibco) supplemented with $10 \%$ fetal bovine serum and penicillin-streptomycin. SARS-CoV-N eukaryotic expression plasmid pDC516N was produced by Dr. Jack Gauldie ${ }^{[11]}$, the University of Ontario, using plasmid pcDNA3.1V5His. Eukaryotic expression vector pcDNA3.1HisA/CXCL16 was produced by our laboratory $^{[2]}$.

\section{Construction of pEGFP-N1/SARS-N}

PCR was carried out with Upstream primer P1: CG
GAATTCATGTCTGATAATGGACCCC (with EcoR I restriction sites) and downstream primer P2: CGGG ATCCCGTGCCTGAGTTGAATC. They were designed and pDC516N was used as a template (with BamH I restriction sites). The amplified fragment was purified by DNA gel extraction kit. The purified product was cloned into pEGFP-N1 to give the Eukaryotic expression vector pEGFP-N1/SARS-N. After digesting by $E c o$ R I and BamH I, the product was ligated by T4 DNA ligase and transformed into DH5 $\alpha$ and the recombinant plasmids were obtained. These positive plasmids were identified by both restriction enzyme digestion and PCR amplification, and sequenced to further confirm the inserted gene.

\section{Construction of pdsRed2-N1/CXCL16}

Upstream primer P3: CGGATTCATGGGACGGG ACTTGC (with EcoR I restriction sites) and downstream primer P4: CGGGATCCCGGGTATTA GAG TCAGGTG (with BamH I restriction sites) were designed and PcDNA3.1HisA/CXCL16 was used as a template. Then the purified product was cloned into pdsRed2-N1 to produce the Eukaryotic expression vector pdsRed2-N1/CXCL16.

\section{Construction of pcDNA3.1HisA/SARS-COVN- GFP}

Upstream primer P3: CGGAATTCATGTCTGATA ATGGACCCC (with EcoR $I$ restriction sites) and downstream primer P2: GCTCTAGACTTGTACAGC TCGTCCATGCC (with $X b a$ I restriction sites) were designed and PEGFP-N1/SARS-COVN was used as a template. Then the purified product was cloned into pcDNA3.1/HisA to construct the Eukaryotic expression vector pcDNA3.1HisA/ SARS-COVN-GFP.

Eukaryotic expression and purification of SARSN-GFP fusion protein 
The recombinant plasmid pcDNA3.1HisA/SARSN-GFP was transfected into HEK293FT cells using the calcium phosphate method. After $48 \mathrm{~h}$ incubation in DMEM containing $10 \%$ fetal calf serum and when fluorescence could be observed under fluorescence microscope, the cells were washed with PBS. After adding protease inhibitors, the cells were processed by ultrasonic cell-break and the supernatant was collected by centrifuging. The proteins were purified with the High-Affinity Ni-NTA Resin and then identified by Western blotting.

\section{Detection of the cellular localization}

Recombinant plasmids pdsRed2-N1/CXCL16 and pEGFP-N1/SARS-N were used to co-transfect HEK293FT cells using the calcium phosphate method. After $48 \mathrm{~h}$ incubation in DMEM containing 10\% fetal calf serum, the tablets were washed once with PBS, fixed with $100 \%$ ethanol, air dried, and observed under the confocal laser scanning microscope.

Recombinant plasmid pdsRed2-N1/CXCL16 was used to transfect HEK293FT cells. After $48 \mathrm{~h}$ incubation in DMEM containing 10\% fetal calf serum the tablets were fixed with $100 \%$ ethanol, washed three times with PBS for five minutes each time. Purified SARS-N-GFP fusion protein was added and after an additional incubation for $1 \mathrm{~h}$ at $37^{\circ} \mathrm{C}$, the tablets were washed three times again for five minutes each time, air dried, and observed under fluorescence microscope.

\section{RESULTS}

\section{Construction and identification of recombinant} expression vector

CXCL16, pDC516N, pcDNA3.1HisA/SARS-NGFP were used as a template, and PCR amplification showed fragments of about $821 \mathrm{bp}, 1,280 \mathrm{bp}, 2,016 \mathrm{bp}$, respectively (Fig.1). After double enzyme digestion, ligation, transformation, colony PCR and restriction endonuclease digestion (Fig. 2) and sequence analysis the positive clones were confirmed. pEGFP-N1/SARSN, pdsRed2-N1/CXCL16, pcDNA3.1HisA/ SARS-NGFP had the correct sequences. The vector was therefore successfully constructed.

Purification and analysis of the expressed protein SARS-N-GFP

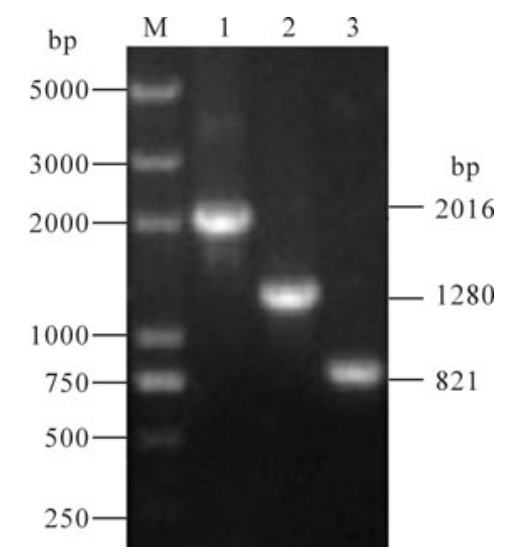

Fig.1. PCR for the SARS-CoV-N-GFP, SRAS-CoV-N, and CXCL16. M, Trans2K Plus DNA Marker; 1, SARS-CoV-NGFP; 2, SARS-CoV-N;3:CXCL16.

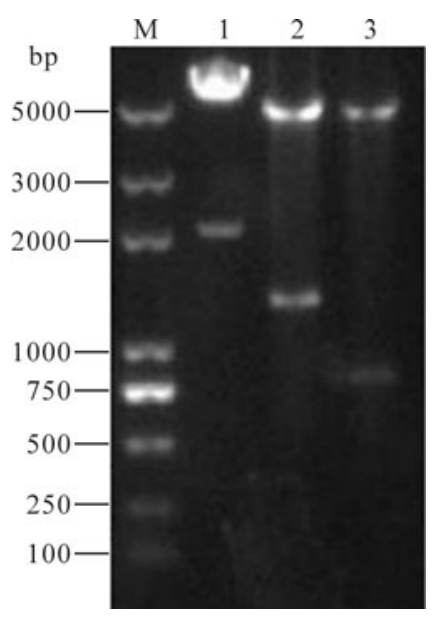

Fig. 2. The recombinant plasmid digested by restriction enzyme. M, Trans2K Plus DNA Marker; 1, pcDNA3.1HisA/ SARSCoV-N-GFP; 2, pEGFP-N1/SARS-CoV-N-GFP; 3, pdsRed2N1/ CXCL16. 
Because of the 6xHis-tag region in the N-terminal of the recombinant SARS-N-GFP protein, SARS-NGFP protein was purified with a High-Affinity Ni NTA Resin. Western blotting was carried out with 6xHIS tag antibody. The purification fusion SARS-NGFP protein was $76 \mathrm{kDa}$ (Fig. 3).

\section{Co-localization of CXCL16 and SARS-N in HEK293FT cells}

pdsRed2-N1/CXCL16 and PEGFP-N1/SARS-N were co-transfected into HEK293FT cells. Confocal laser scanning microscopy was employed to establish the location of these proteins within the cells. The CXCL16, which showed red fluorescence was mainly located in the cell membrane and cytoplasm; SARS-N, showing green fluorescence was mainly located in the cytoplasm Co-location of the two kinds of protein in the cytoplasm showed an orange fluorescence (Fig. 4).

Excellular combination and location of SARS-N with the transfected CXCL16

pdsRed2-N1/CXCL16 was used to transfect HEK293FT cells, and purified SARS-N-GFP fusion protein was added. Fluorescence microscopy was employed to show the position of the proteins. After $1 \mathrm{~h}$ incubation at $37^{\circ} \mathrm{C}, \mathrm{CXCL} 16$ showed a red fluorescence, and attached SARS-N-GFP showed green fluorescence on the membrane of the transfected HEK293FT cells.

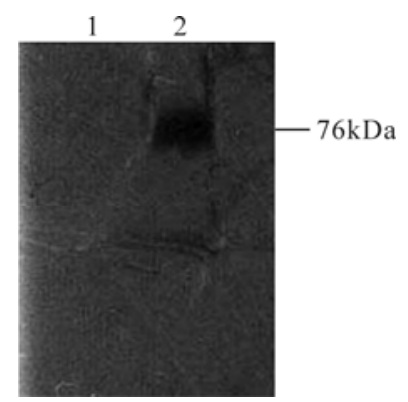

Fig. 3. Western blotting for SARS-CoVN-GFP protein. 1, 293FT tansfection with pcDNA3.1/ HisA(negative control); 2, Purified SARS- CoVN-GFP protein.
This changed to orange fluorescence when the two areas overlapped (Fig. 5).

\section{DISCUSSION}

CXCL16 is a newly discovered cell chemokine, composed of 254 amino acids, with a relative molecular mass of about $30,000 \mathrm{Da}^{[14]}$. CXCL16 with chemotactic function exists in the membrane-bound form or secreted form. CXCL16 produces a strong chemotaxis for activated $\mathrm{CD}^{+} \mathrm{T}$ cells, and can also promote the interaction between antigen-presenting cells (APC) and T cells, induce the immune response of specific cell subsets, and regulate the migration of activated $\mathrm{T}$ cells in the spleen red pulp ${ }^{[10]}$. In addition, with the application of CXCL16 monoclonal antibodies, it was discovered that tonsil and CD19+B cells could express the chemokine, indicating that CXCL16 were involved in the cell-cell interactions in chronic inflammation. The only CXCL16 receptor was named CXCR6, and CXCR6-positive T cells play a very important role in some particular diseases ${ }^{[13]}$. SARS$\mathrm{CoV} \mathrm{N}$ protein is released to the outside of the cell and combined with CXCL16, which may prevent or reduce the chemotactic function of CXCL16, and in turn immune cells could not gather at the site of SARS-CoV infection and play a functional role in immune response. The combination of SARS-CoV N protein and membrane-bound CXCL16 might assist the host cell endocytosis to SARS-CoV virus. Studies have shown that the mucosal surface is important in HIV invasion, and high CXCL16 expression in the mucous membrane areas may affect the ability of HIV infective particle to use the CXCL16 receptor as an assistant receptor ${ }^{[8]}$. SARS-CoV N protein is also likely to play the role of a viral chemokine-binding protein. 


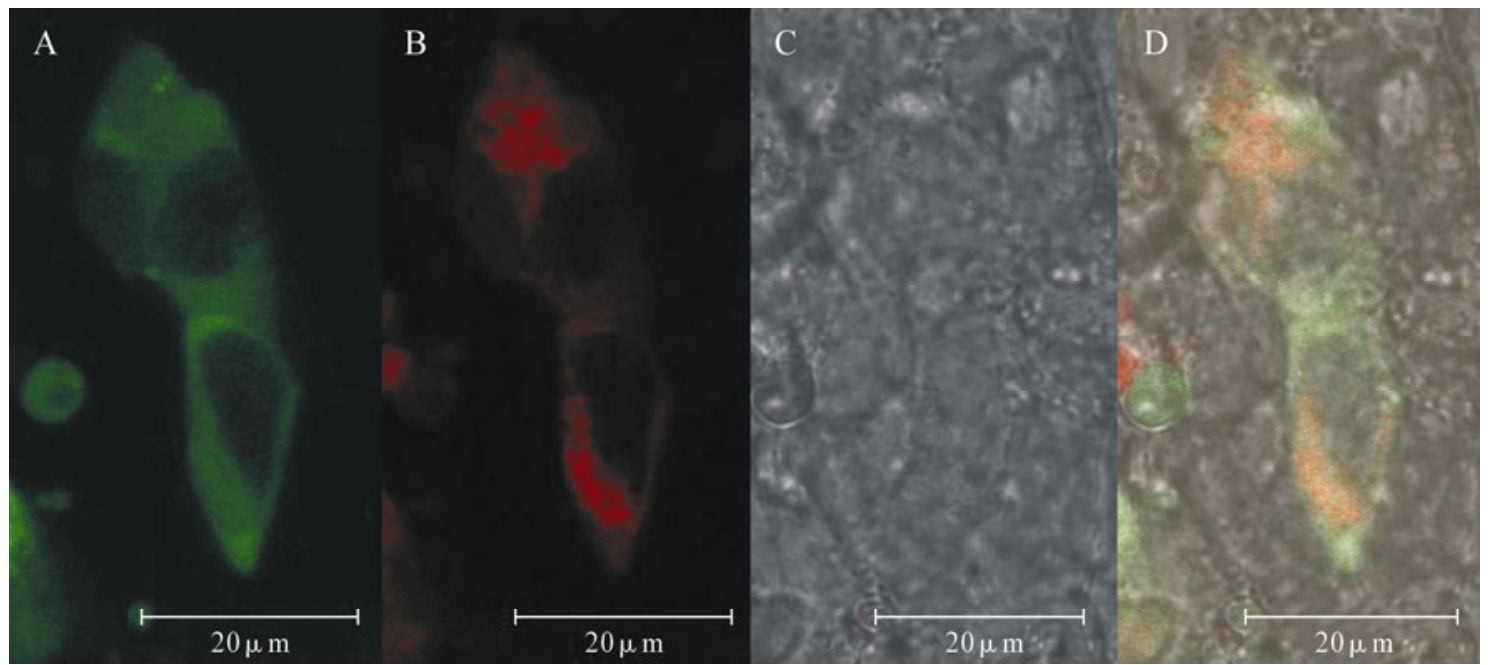

Fig. 4. N protein of SARS-CoV interacting with CXCL16 in cell. At $48 \mathrm{~h}$ post transfection cells were visualized with a laser confocal microscope. A: The positive 293FT cells transfected with pEGFP-N1/SARS-CoVN and PRFP fluorescently labeled SARS-N protein 293FT cells (UV:488nm). B: transfected with pdsRed2-N1/CXCL16 were visulized by a confocal microscopy (UV:558nm). C: The same cells were checked with white light. D: The yellow cells were also under double wave UV.

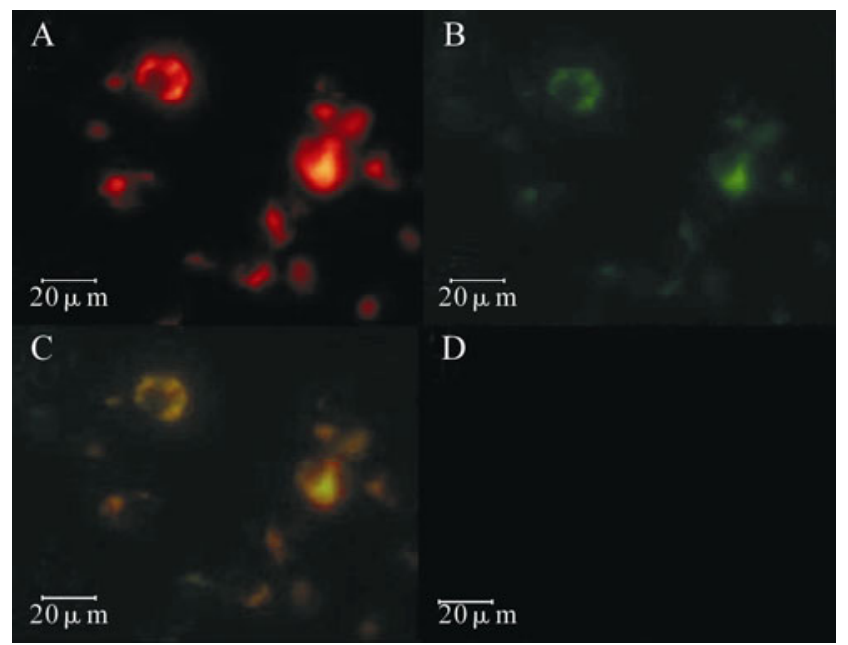

Fig. 5. N protein of SARS-CoV interacting with CXCL16 out cell. At $48 \mathrm{~h}$ post transfection the 293FT cells pdsRed2-N1/ CXCL16 were fixed and stained with pure GFP labeled SARS-N protein. A: The red cells were visualized with a fluoresce microscope (UV558nm).B: The green light was visualized around some cell membrane (UV488 nm). C: The yellow cell were visualized when A and B were merged. D:The 293FT cells transfected with pdsRed2-N1 and stained with SARS-CoVN-GFP protein (UV488 nm).

Studies have shown that some viruses can synthesize viral chemokine-binding protein, which can be combined with a variety of chemokines and results in their loss of chemotactic function. For example, myxoma virus can synthesize the viral chemokinebinding protein M-T7. It can inhibit the chemotaxis signals required in the monocyte/M $\Phi$ flow, that is, it can integrate with and inhibit CC chemokines. Lalani and others first proved that the purified M-T7 can combine with $\mathrm{CXC}, \mathrm{CC}$ and $\mathrm{C}$ chemokines, and this combination is non-species- specific ${ }^{[6]}$. Purified M-T7 protein can inhibit infection and blood cell recruitment and activate intimal hyper- plasia which is involved by the inflammatory site. 
In this study, the eukaryotic expression vector was constructed and the recombinant fusion protein SARS-CoVN with GFP and recombinant protein CXCL16 with RFP were co-expressed in HEK293FT cells. Through confocal laser scanning microscopy, it was observed that CXCL16 showed red fluorescence, and was mainly localized in the cell membrane and cytoplasm; SARS-CoV N, mainly localized in the cytoplasm, showed green fluorescence, in similar locations. The co-localization of the two kinds of protein in the cytoplasm showed orange fluorescence. In our experiment, a eukaryotic expression vector pcDNA3.1HisA/SARS-CoVN-GFP was also constructed and transfected into HEK293FT cells. A HIS-tagged fusion protein SARS-CoVN-GFP was expressed, purified through the High-Affinity Ni-NTA Resin, and incubated in vitro with HEK293FT cells which had been transfected with pcDNA3.1HisA/CXCL16. Under a fluorescence microscope, it could be seen the SARSCoVN-GFP fusion protein and the CXCL16-RED protein were co-localized in the cell membrane, which confirmed the two could interact with each other.

\section{Reference}

1. Brunn A A, Teepe C, Simpson J C, et al. 2007. Analysis of intraviral protein-protein interactions of the SARS coronavirus ORFeome. PLoS ONE, 23: 459.

2. Chang W S, Zhai J, Song W G. 2008. Screen and Identification of The Protein-protein Interactors in The HostCell With The SARS Coronavirus Nucleocapsid Protein. Prog Biochem Biophy, 35 (9): 1007-1013. (in Chinese)

3. Fan Z, Zhou Y, Tan X, et al. 2006. SARS-CoV nucleocapsid protein binds to hUbc9, a ubiquitin conjugating enzyme of the sumoylation system. J Med Virol, 78: 1365-1373.

4. Kopecky-Bromberg S A, Martínez-Sobrido L, Frieman M, et al. 2007. Severe acute respiratory syndrome coronavirus open reading frame (ORF) $3 \mathrm{~b}$, ORF 6 , and nucleocapsid proteins function as interferon antagonists. $\mathbf{J}$ Virol, 81: 548-557.

5. Lalani A S, Graham K, Mossman K, et al. 1997. The purified myxoma virus gamma interferon receptor homolog M-T7 interacts with the heparin domains of chemokines. J Virol, 71 (6): 4356-4363.

6. Luo C, Luo H, Zheng S, et al. 2004. Nucleocapsid protein of SARS coronavirus tightly binds to human cyclophilin A, Biochem Biophys Res Commun, 321: 557-565.

7. Luo H, Chen Q, Chen J, et al. 2005. The nucleocapsid protein of SARS coronavirus has a high binding affinity to the human cellular heterogeneous nuclear ribonucleoprotein A1, FEBS letters, 579: 2623-2628.

8. Matloubian M, David A, Engel S, et al. 2000.A transmembrane CXC chemokine is a ligand for HIVcoreceptor Bonzo. Nat Immunol, 1 (4): 298-304.

9. Saikatendu K S, Jeremiah J S, Subramanian V, et al. 2007. Ribonucleocapsid Formation of Severe Acute Respiratory Syndrome Coronavirus through Molecular Action of the N-Terminal Domain of N Protein. J Virol, 81: 3913-3921.

10. Shashkin P, Simpson D, Mishin V, et al. 2003. Expression of CXCL16 in human T cells. Arterioscler Thromb Vasc Biol, 23 (1): 148-149.

11. Surjit M, Kumar R, Mishra R N, et al. 2005. The Severe Acute Respiratory Syndrome Coronavirus Nucleocapsid Protein Is Phosphorylated and Localizes in the Cytoplasm by 14-3-3-Mediated Translocation,J Virol, 79: 11476-11486.

12. Surjit M, Liu B, Chow V T, et al. 2006. The Nucleocapsid Protein of Severe Acute Respiratory Syndrome-Coronavirus Inhibits the Activity of CyclinCyclin-dependent Kinase Complex and Blocks S Phase Progression in Mammalian Cells. J Biol Chem, 281: 10669-10681.

13. Wei W J, Wen H Y. 2007. The role of Chemokine CXCL16 in clinic diseases. Prog Modern Biomed, 7 (5): 798-800. (in Chinese).

14. Wilbanks A, Zondlo S C, Murphy K, et al. 2001. Expression cloning of the STRL33/BONZO/TYMSTR ligand reveals elements of $\mathrm{CC}, \mathrm{CXC}$, and $\mathrm{CX} 3 \mathrm{C}$ chemokines. J Immunol, 166 (8): 5145-5154.

15. Zhao X G, Nicholls J M, Chen Y G. 2007. Sars-cov nucleocapsid protein interacts with smad 3 and modulates TGF- $\beta$ signaling. J Biol Chem, 283: 3272-3280. 